\title{
Pathogenesis of Diabetic Neuropathy and the Treatment Efforts for it
}

\author{
Tarek Elshourbagy* \\ Cairo University, Egypt \\ *Corresponding Author: Tarek Elshourbagy, Cairo University, Egypt.
}

Received: July 12, 2019; Published: July 25, 2019

DOI: 10.31080/ASNE.2019.02.0089

\begin{abstract}
Neuropathy is the commonst complication in DM. Prolonged hyperglycemia is the cause of metabolic changes which leads to peripheral nerve injury through increased polyol influx, enhanced advanced glycation end products formation, oxidative stress and exaggerated release of inflammatory cytokines. In this article we will discuss these mechanisms and the treatment efforts targeting them.

Keywords: Neuropathy; Diabetic Neuropathy
\end{abstract}

\section{Introduction}

Peripheral neuropathy is the commonst complication of DM $[1,2]$. It involves sensory, motor and autonomic nerves. Multiple studies showed that autonomic cardiovascular neuropathy dramatically shortens the patients lifespan and increases mortality $[3,4]$. Without exploring the exact causative factors involved in diabetic neuropathy, it will not be possible to develop an effective treatment for it. Unfortunately, there is no current effective treatment for diabetic neuropathy at a global level.

Pathogenesis of peripheral neuropathy in diabetes mellitus

The polyol pathway

Increased polyol influx regulated by the enzyme Aldose Reductase has been studied extensively and it is considered one of the main mechanisms involved in peripheral neuropathy.

Multiple drugs have been developed targeting Aldose Reductase. However, they have serious adverse effects and or there was no significant improvement during clinical trials. Epalrestat is an Aldose Reductase Inhibitors(ARIs) which showed marked improvement only in patients with early neuropathy and modestly elevated levels of glycated haemoglobin [5].

\section{Glycation and AGE products}

Glycation has been involved in the pathogenesis of diabetic neuropathy [6-8]. Deposition of AGEs was shown in animal and human diabetic neurons; Stromal collagen, axoplasms of nerve fibers, schwann cells and endoneuorial vessels [9] were affected by glycation and AGEs, schwann cells underwent apoptosis with release of TNF alpha and other inflammatory cytokines when ex-

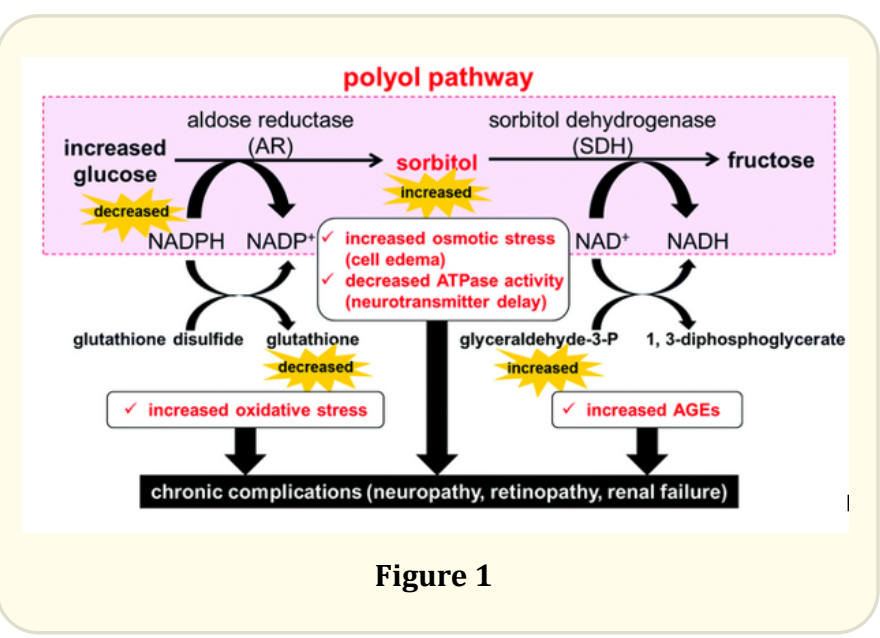

posed to a high AGEs environment [10]; axonal cytoskeleton of tubulin and neurofilaments caused stagnation of axonal transport leading to distal nerve fiber degeneration [6]; glycation of basement membrane collagen, laminin and fibronectin caused impairment of nerve fiber regeneration.

Currently, there is are a few agents can be administered acting on this mechanism such as: Atrovastatin which is found to reduce AGE formation through its anti-oxidative activity. Another agent is sRage which is found to completely inhibit the receptors for AGE known as RAGE inhibitors [11].

\section{Oxidative stress}

Generation of reactive oxygen species(ROS) is considered a major factor contributing to the development of diabetic neuropathy $[12,13]$; many experiments showed that oxidative stress can cause nerve fiber injury [14-17]. 


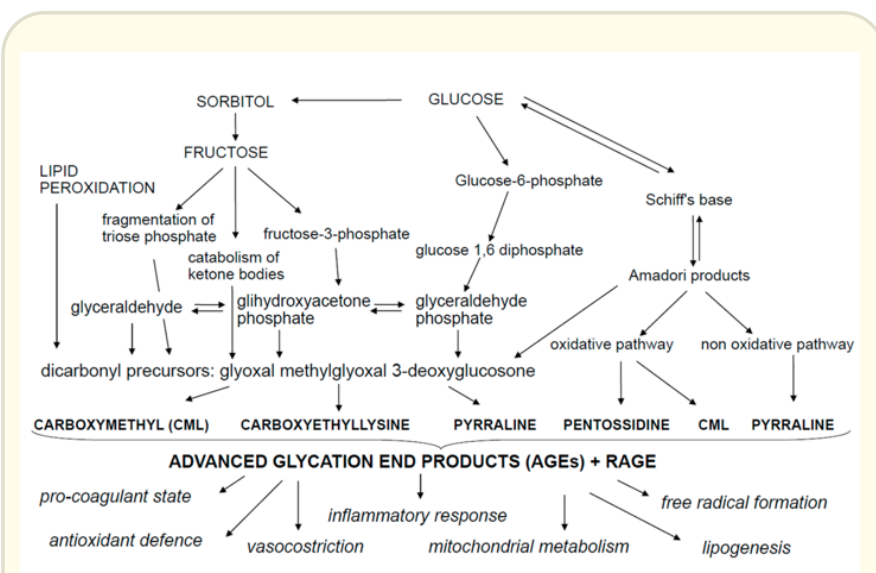

Figure 2

There are multiple attempts to inhibit diabetic neuropathy with using Antioxidants. One of these drugs is Alpha lipolic acid. In vivo it showed improvement in nerve conduction velocity delay, nerve blood flow and nerve structure [18,19].

Administration of Vitamin E resulted in reversal of defective nerve conduction in patients with mild to moderate diabetic Neuropathy [20].

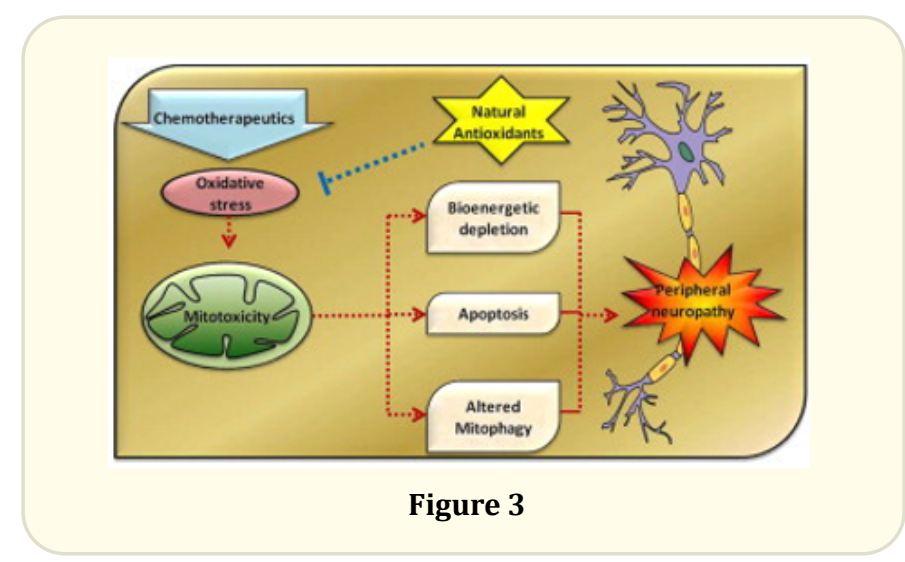

\section{Exaggeration of proinflammatory cytokines release}

Numerous studies showed that neurons undergo a proinflammatory process which cause symptoms and aggravates the development of neuropathy [21,22]; Diabetic neurons contain macrophages and there is increased release of TNF alpha and Interleukins(ILs) [21,23,24].

Currently, numerous studies are carried out targeting cytokines to overcome diabetic neuropathy; drugs as Pioglitazone and N- acetyl cycteine showed improvement of nerve conduction velocity delay in STZ-diabetic rats $[25,26]$. Through inhibition of cytokines release or macrophages migration.

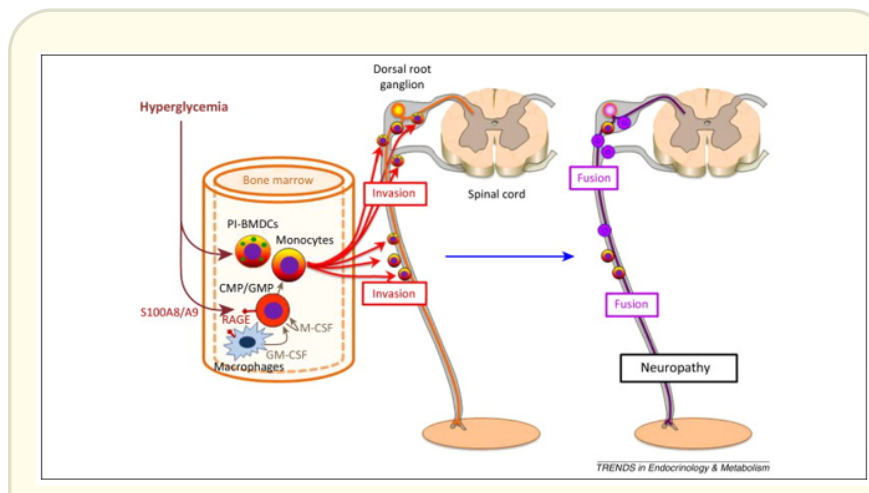

Figure 4

\section{Conclusion}

Diabetic neuropathy is a serious disorder and the mechanisms beyond it are complex. We have discussed four main mechanisms and the treatment efforts acting on them. More studies should be carried out to investigate deeply in the mechanisms beyond Diabetic Neuropathy to be able to develop more efficient drugs with less adverse effects.

\section{Bibliography}

1. Boulton AJ., et al. "Diabetic neuropathies: a statement by the American Diabetes Association”. Diabetes Care 28 (2005): 956962.

2. Strotmeyer ES., et al. "Sensory and motor peripheral nerve function and lower-extremity quadriceps strength: the health, aging and body composition study". Journal of the American Geriatrics Society 57 (2009): 2004-2010.

3. Ewing DJ., et al. "Mortality in diabetic autonomic neuropathy". Lancet 1 (1976): 601-603.

4. Ewing DJ., et al. "The natural history of diabetic autonomic neuropathy". QJM 49 (1980): 95-108.

5. Hotta N., et al. "Stratified analyses for selecting appropriate target patients with diabetic peripheral neuropathy for longterm treatment with an aldose reductase inhibitor, epalrestat". Diabetic Medicine 25 (2008): 818-825. 
6. Yagihashi S. "The pathogenesis of diabetic neuropathy". Diabetes/Metabolism Research and Reviews 11 (1995): 193-225.

7. Thornalley PJ. "Glycation in diabetic neuropathy: characteristics, consequences, causes, and therapeutic options". International Review of Neurobiology 50 (2002): 37-57.

8. Sugimoto K., et al. "Role of advanced glycation end products in diabetic neuropathy". Current Pharmaceutical Design 14 (2008): 953-961.

9. Sugimoto K., et al. "Localization in human diabetic peripheral nerve of $\mathrm{N}$ (epsilon)-carboxymethyllysine-protein adducts, an advanced glycation end product". Diabetologia 40 (1997): 1380-1387.

10. Sekido H., et al. "Reduced cell replication and induction of apoptosis by advanced glycation end products in rat Schwann cells". Biochemical and Biophysical Research Communications 320 (2004): 241-248

11. "The role of Advanced Glycation End Products in Diabetic Vascular Complications". Sang Yool Rhee and Young Seol Kim.

12. Greene DA., et al. "Glucose-induced oxidative stress and programmed cell death in diabetic neuropathy". European Journal of Pharmacology 375 (1999): 217-223.

13. Pop-Busui R., et al. "Diabetic neuropathy and oxidative stress". Diabetes/Metabolism Research and Reviews 22 (2006): 257273.

14. Stevens MJ., et al. "C-peptide corrects endoneurial blood flow but not oxidative stress in type $1 \mathrm{BB} /$ Wor rats". American Journal of Physiology-Endocrinology and Metabolism 287 (2004): E497-E505.

15. Obrosova IG., et al. "High-fat diet induced neuropathy of prediabetes and obesity: effects of "healthy" diet and aldose reductase inhibition". Diabetes 56 (2007): 2598-2608.

16. Bierhaus A., et al. "Loss of pain perception in diabetes is dependent on a receptor of the immunoglobulin superfamily". Journal of Clinical Investigation 114 (2004): 1741-1751.

17. Wada R., et al. "Effects of OPB-9195, anti-glycation agent, on experimental diabetic neuropathy". European Journal of Clinical Investigation 31 (2001): 513-520.
18. Cameron NE., et al. "Anti-oxidant treatment prevents the development of peripheral nerve dysfunction in streptozotocindiabetic rats". Diabetologia 36 (1993): 299-304.

19. Stevens MJ., et al. "Effects of DL-alpha-lipoic acid on peripheral nerve conduction, blood flow, energy metabolism, and oxidative stress in experimental diabetic neuropathy". Diabetes 49 (2000): 1006-1015.

20. N B Tütüncü., et al. "Reversal of defective nerve conduction in with Vitamin E supplementation in type 2 diabetes mellitus: a preliminary study". 21.11 (1998): 1915-1918.

21. Younger DS., et al. "Diabetic peripheral neuropathy: a clinicopathologic and immunohistochemical analysis of sural nerve biopsies". Muscle Nerve 19 (1996): 722-727.

22. Satoh J., et al. "The possible role of tumor necrosis factor-alpha in diabetic polyneuropathy". Archive of Experimental Diabesity Research 4 (2003): 65-71.

23. Conti G., et al. "Macrophage infiltration and death in the nerve during the early phases of experimental diabetic neuropathy: a process concomitant with endoneurial induction of IL-1beta and p75NTR". Journal of the Neurological Sciences 195 (2002): $35-40$.

24. Yagihashi S., et al. "Pathology and pathogenetic mechanisms of diabetic neuropathy: correlation with clinical signs and symptoms". Diabetes Research and Clinical Practice 77 (2007): S184-S189.

25. Sagara M., et al. "Inhibition of development of peripheral neuropathy in streptozotocin-induced diabetic rats with $\mathrm{N}$-acetylcysteine". Diabetologia 39 (1996): 263-269.

26. Yamagishi S., et al. "Correction of protein kinase $\mathrm{C}$ activity and macrophage migration in peripheral nerve by pioglitazone, peroxisome proliferator activated-gamma-ligand, in insulindeficient diabetic rats". Journal of Neurochemistry 104 (2008): 491-499.

Volume 2 Issue 8 August 2019

(C) All rights are reserved by Tarek Elshourbagy. 